\title{
Frequency Domain Heart Period and QT Interval Variability Markers Are Linked to Arrhythmic Risk in Long QT Syndrome Type 2
}

\author{
Vlasta Bari ${ }^{1}$, Giulia Girardengo ${ }^{2}$, Beatrice De Maria ${ }^{3}$, Beatrice Cairo ${ }^{4}$, Lia Crotti ${ }^{2,5,6}$, \\ Peter J Schwartz ${ }^{5}$, Alberto Porta ${ }^{1,4}$ \\ ${ }^{1}$ Department of Cardiothoracic, Vascular Anesthesia and Intensive Care, IRCCS Policlinico San \\ Donato, San Donato Milanese, Milan, Italy \\ ${ }^{2}$ IRCCS Istituto Auxologico Italiano, Department of Cardiovascular, Neural and Metabolic Sciences, \\ San Luca Hospital, Milan, Italy \\ ${ }^{3}$ IRCCS Istituti Clinici Scientifici Maugeri, Milan, Italy \\ ${ }^{4}$ Department of Biomedical Sciences for Health, University of Milan, Milan, Italy \\ ${ }^{5}$ IRCCS Istituto Auxologico Italiano, Center for Cardiac Arrhythmias of Genetic Origin, Milan, Italy \\ ${ }^{6}$ Department of Medicine and Surgery, University of Milano-Bicocca, Milan, Italy
}

\begin{abstract}
Long QT syndrome type 2 (LQT2) is an inherited disease leading to arrhythmias and sudden death often in response to an abrupt sympathetic stimulus. Heart period (HP) and $Q T$ interval variability analyses were carried out over asymptomatic (ASYMP, $n=10$, age 39.0 11.0 yrs, 9 males) and symptomatic (SYMP, $n=7$, age $41.7 \pm 12.8$ yrs, 4 males) LQT2 patients. Beat-to-beat series were derived from $24 \mathrm{~h}$ Holter ECG recordings during daytime and nighttime. In addition to HP and $Q T$ mean and variance, we calculated HP power in high frequency ( $\mathrm{HF}$, from 0.15 to $0.5 \mathrm{~Hz}$ ) band as a marker of vagal modulation directed to sinus node and QT power in low frequency ( $L F$, from 0.04 to $0.15 \mathrm{~Hz}$ ) band as a marker of sympathetic modulation directed to ventricles. $H P$ and $Q T$ means exhibited significant day-night changes in both groups. ASYMP showed a shorter corrected $Q T$ and a more reactive vagal modulation leading to significant day-night variations of respiratory sinus arrhythmia. By contrast, SYMP had no significant day-night variations of the vagal control. Conversely, SYMP showed a higher sympathetic control during day compared to ASYMP, having a larger $Q T$ variance. These findings suggest that frequency domain $H P$ and $Q T$ variability markers can account for the different arrhythmic risk of LQT2 ASYMP and SYMP patients.
\end{abstract}

\section{Introduction}

The long QT syndrome (LQTS) is an inherited genetic disease affecting cardiac cell ion channels and whose main clinical sign is the prolongation of the QT interval on the surface electrocardiogram (ECG) [1]. It has often dramatic manifestations, such as life-threatening arrhythmias and sudden death. LQTS type 2 (LQT2) is the second main variant of LQTS, accounting for $35-40 \%$ of the overall LQTS population [2]. LQT2 is associated with a mutation on the $\mathrm{KCNH} 2$ gene influencing the rapid component $\mathrm{I}_{\mathrm{Kr}}$ of the delayed rectifier potassium current $\mathrm{I}_{\mathrm{K}}$ [3]. Symptoms of LQT2 consequent to cardiac arrhythmias are more likely to occur in response to an abrupt sympathetic challenge, such as a sudden emotional stress or an auditory stimulus, as the alarm clock or a telephone ringing in the middle of the night $[1,4]$. Moreover, symptoms are more likely to occur at awakening time. A peculiarity of all the forms of LQTS is the presence of individuals, possibly siblings, featuring the same mutation but with two completely different phenotypes, respectively, with no symptoms and limited arrhythmic risk or with major symptoms and at the greatest risk of fatal events [2]. The two classes are usually denoted as symptomatic (ASYMP) and symptomatic (SYMP).

In LQTS type 1 (LQT1), the main variant of LQTS, the sympathetic nervous system plays a very important role in triggering arrhythmias [5] and in modulating the arrhythmic risk [6]. In analogy with LQT1, it can be hypothesized that there is an association between the arrhythmic risk of LQT2 patients and cardiac autonomic profile. Thus, the aim of this study was to characterize cardiac autonomic control of LQT2 patients subdivided into ASYMP and SYMP individuals via time and frequency domain markers computed over HP and QT beat-to-beat variability [7-10]. HP and QT series were extracted from 24h Holter ECG recordings. 

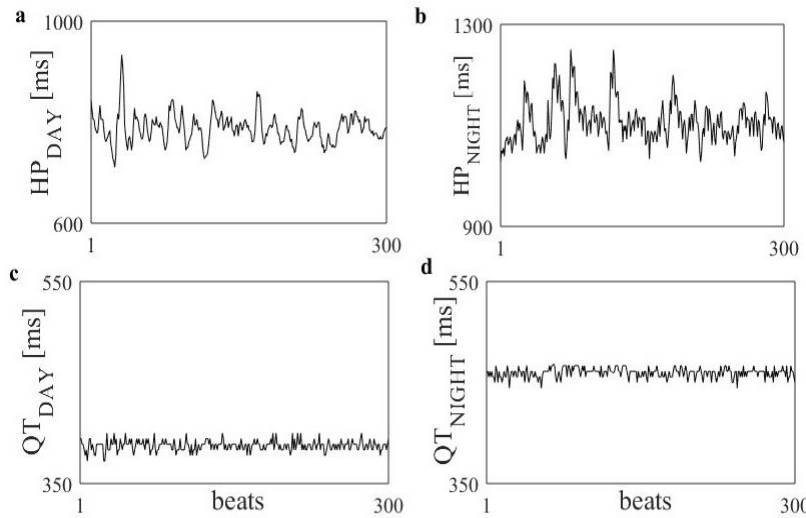

Figure 1. Examples of HP (a,b) and QT $(c, d)$ time series extracted from an ASYMP subject during DAY (a,c) and NIGHT $(b, d)$.

\section{Materials and Methods}

\subsection{Data Acquisition}

Ten ASYMP subjects (age: 39.0 $\pm 11.0,9$ males) and 7 SYMP subjects (age 41.7 $\pm 12.8,4$ males) were enrolled in this study. From each patient a 12 lead 24h Holter ECG recording was acquired (Mortara Instruments Inc, Milwakee, WI) with a sampling rate of $180 \mathrm{~Hz}$. We selected the lead with the best signal-to-noise ratio. The study was performed in keeping with the principles of Declaration of Helsinki for studies involving human subjects and the protocol was approved by the ethical committee in charge for University of Pavia, Pavia, Italy and IRCCS Istituto Auxologico Italiano, Milan, Italy. All participants signed an informed consent.

\subsection{Experimental Protocol}

From the ECG, HP was extracted as the time distance between two consecutive R-wave peaks. Jitters of the location of the R-wave apex were minimized via parabolic interpolation. QT interval was approximated as the time distance between the second R-peak delimiting the current HP and the end of the T wave. The T-wave offset was located as the point where the first derivative calculated on the downslope of the $\mathrm{T}$ wave reached the $30 \%$ of its maximum [11]. HP and QT series detections were visually inspected, checked and, then, corrected via cubic spline interpolation if measures were the result of ectopic beats or misdetections. We corrected a maximum of the $5 \%$ of the total length of the extracted series. Two epochs lasting 5000 beats were extracted from each $24 \mathrm{~h}$ Holter ECG recording, one during daytime (DAY, from 2:00 to 6:00 p.m.) and one during nighttime (NIGHT, from 12:00 to 4:00 a.m.).
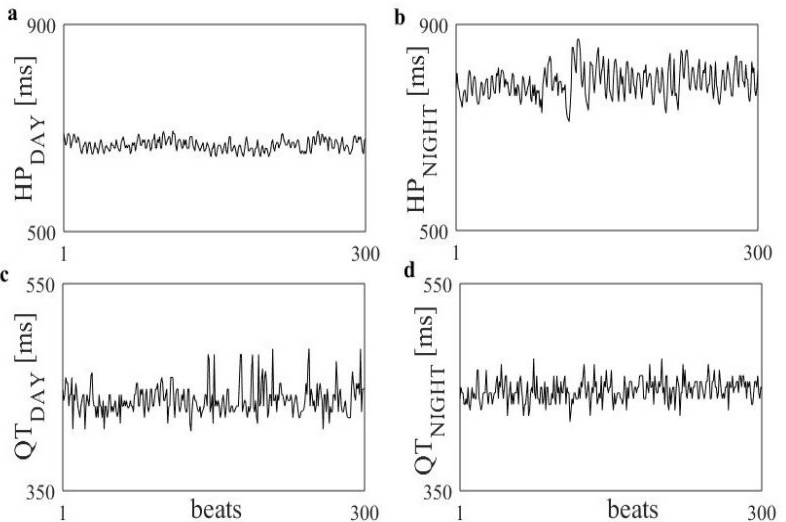

Figure 2. Examples of HP $(a, b)$ and QT $(c, d)$ time series extracted from a SYMP subject during DAY $(a, c)$ and NIGHT $(b, d)$.

\subsection{Data Analysis}

Analysis was carried out over 300 consecutive measures and iterated with $50 \%$ overlap between adjacent frames over the overall considered segment (i.e. 5000 beats). Figure 1 shows an example of a HP series of 300 measures extracted during DAY (Fig.1a) and NIGHT (Fig.1b) and of a QT beat-to-beat series extracted as well during DAY (Fig.1c) and NIGHT (Fig.1d) in an ASYMP subject. Figure 2 has the same structure of Fig.1 but it shows HP and QT series in a SYMP subject. The median of the distribution of the computed markers over the entire segment was taken as representative of the subject and underwent additional statistical analysis.

Mean and variance of HP and QT interval were calculated and labeled as $\mu_{\mathrm{HP}}, \mu_{\mathrm{QT}}, \sigma_{\mathrm{HP}}^{2}, \sigma_{\mathrm{QT}}^{2}$. Indexes were respectively expressed in $\mathrm{ms}, \mathrm{ms}, \mathrm{ms}^{2}$ and $\mathrm{ms}^{2}$. Then, series were linearly detrended and power spectral density was calculated. The series were described via an autoregressive model [12]. The coefficients of the model were estimated via the Levinson-Durbin recursion and the model order was optimized via the Akaike information criterion. Power spectral density was decomposed into frequency components [13]. Each component was labeled as low frequency (LF, from 0.04 to $0.15 \mathrm{~Hz}$ ) or high frequency (HF, from 0.15 to $0.5 \mathrm{~Hz}$ ) according to its central frequency. The power of HP in HF band was taken as a marker of vagal modulation directed to the sinus node [7], while the power of QT in LF band was taken as an index of sympathetic modulation directed to the ventricles $[8,14]$. Both indexes were expressed in $\mathrm{ms}^{2}$ and labeled respectively as $\mathrm{HF}_{\mathrm{HP}}$ and $\mathrm{LF}_{\mathrm{QT}}$.

\subsection{Statistical Analysis}

Two-way repeated measures analysis of variance (one factor repetition, Holm-Sidak test for post hoc multiple comparisons) was applied to assess statistical differences 
Table 1. HP and QT time and frequency domain indexes in LQT2 SYMP and ASYMP groups during DAY and NIGHT.

\begin{tabular}{ccccc}
\hline \multirow{2}{*}{ Parameter } & \multicolumn{2}{c}{ DAY } & ASYMP & SYMP \\
\cline { 2 - 5 } & ASYMP & SYMP & $1084.3 \pm 128.8^{*}$ & $938.5 \pm 153.8^{*}, \S$ \\
$\mu_{\mathrm{HP}}[\mathrm{ms}]$ & $891.2 \pm 96.6$ & $756.8 \pm 81.5^{\S}$ & $3182.1 \pm 2665.4$ \\
$\sigma^{2}{ }_{\mathrm{HP}}\left[\mathrm{ms}^{2}\right]$ & $3318.4 \pm 2615.1$ & $2256.5 \pm 1540.8$ & $5031.5 \pm 3605.6$ & $404.5 \pm 237.5$ \\
$\mathrm{HF}_{\mathrm{HP}}\left[\mathrm{ms}^{2}\right]$ & $375.2 \pm 331.6$ & $169.4 \pm 149.1$ & $981.8 \pm 1001.9^{*}$ & $403.5 \pm 37.7^{*}$ \\
$\mu_{\mathrm{QT}}[\mathrm{ms}]$ & $381.9 \pm 25.4$ & $374.0 \pm 57.2$ & $422.0 \pm 34.8^{*}$ & $204.2 \pm 229.0$ \\
$\sigma_{\mathrm{QT}}^{2}\left[\mathrm{~ms}^{2}\right]$ & $102.9 \pm 84.4$ & $298.0 \pm 247.0^{\S}$ & $87.6 \pm 46.8$ & $36.9 \pm 37.7$ \\
LF $_{\mathrm{QT}}\left[\mathrm{ms}^{2}\right]$ & $19.5 \pm 17.9$ & $37.3 \pm 19.1$ & $16.4 \pm 9.5$ & \\
\hline
\end{tabular}

$\mu_{\mathrm{HP}}=\mathrm{HP}$ mean; $\sigma_{\mathrm{HP}}^{2}=\mathrm{HP}$ variance; $\mathrm{HF}_{\mathrm{HP}}=$ power of $\mathrm{HP}$ series in $\mathrm{HF}$ band; $\mu_{\mathrm{QT}}=\mathrm{QT}$ mean; $\sigma^{2} \mathrm{QT}=\mathrm{QT}$ variance; $\mathrm{LF}_{\mathrm{QT}}=$ power of QT series in LF band; ASYMP=asymptomatic subjects; SYMP=symptomatic subjects; DAY=daytime; NIGHT $=$ nighttime. Results are reported as mean \pm standard deviation. The symbols $*$ and $\S$ indicate, respectively, $p<0.05$ versus DAY within the same group (i.e. ASYMP or SYMP) and versus ASYMP within the same period (i.e. DAY or NIGHT).

between ASYMP and SYMP within the same period of analysis (i.e. DAY or NIGHT) and between DAY and NIGHT within the same group (i.e. ASYMP or SYMP). A $p<0.05$ was always deemed as significant.

\section{Results}

Table 1 shows time and frequency domain indexes derived from HP and QT series in ASYMP and SYMP subjects. The parameter $\mu_{\mathrm{HP}}$ was longer during NIGHT compared to DAY in both groups. In agreement with the notion that HP lengthening is protective in LQT2, $\mu_{\mathrm{HP}}$ was longer in ASYMP than in SYMP subjects during both DAY and NIGHT. $\sigma^{2}$ HP was unchanged between groups and conditions. $\mathrm{HF}_{\mathrm{HP}}$ was increased in ASYMP subjects during NIGHT compared to DAY, while no DAY-NIGHT variations were observed in SYMP ones. $\mathrm{HF}_{\mathrm{HP}}$ was not able to separate groups within the same period of analysis (i.e. DAY or NIGHT). The marker $\mu_{\mathrm{QT}}$ was increased in both groups during NIGHT with respect to DAY but it did not distinguish the two groups either during DAY or NIGHT. The parameter $\sigma^{2}{ }_{\mathrm{QT}}$ was higher in SYMP subjects than in ASYMP ones during DAY, while the two groups did not differ during NIGHT. The marker $\sigma^{2}{ }_{\mathrm{QT}}$ did not exhibit circadian variations in both groups. $\mathrm{LF}_{\mathrm{QT}}$ did not vary between groups and conditions.

\section{Discussion}

Our main findings are: i) circadian variations of HP and QT means are preserved in LQT2 subjects; ii) LQT2 ASYMP subjects have a longer HP during both DAY and NIGHT; iii) HF power of HP increases in LQT2 ASYMP during NIGHT, while this increment is not visible in SYMP, thus suggesting a more reactive vagal control in ASYMP; iv) QT variance is higher in LQT2 SYMP subjects than in ASYMP ones during DAY, thus suggesting a more active sympathetic control in SYMP group.
Both ASYMP and SYMP showed preserved circadian variations of the $\mathrm{HP}$ and QT means. In addition, in the ASYMP group, HP mean was longer in both DAY and NIGHT than in SYMP subjects. Remarkably, QT mean was similar in ASYMP and SYMP groups. The longer HP in ASYMP subjects, together with the similar values of QT, led to a shorter corrected QT in ASYMP individuals. These characteristics of the ASYMP group confirm the well-known notion that longer HP and shorter corrected QT interval are protective factors against the development of arrhythmias in LQT2 [15].

Moreover, LQT2 ASYMP group showed a more reactive vagal control as assessed via the magnitude of the respiratory sinus arrhythmia (i.e. $\mathrm{HF}_{\mathrm{HP}}$ ) compared to SYMP: indeed, $\mathrm{HF}_{\mathrm{HP}}$ significantly increased during NIGHT in ASYMP, while it remained unmodified in SYMP subjects. We hypothesize that SYMP patients have a lower capacity to modulate HP through vagallymediated neural inputs and this situation would make this group more prone to develop arrhythmias.

On the contrary, SYMP subjects were characterized by an increased QT variance with respect to ASYMP ones during DAY, thus suggesting a higher sympathetic control directed to ventricles [8-10]. This greater sympathetic drive might be responsible for the higher heart rates of SYMP subjects that expose them to a greater arrhythmic risk when an additional abrupt sympathetic challenge would require a further and sudden shortening of QT $[1,2,4]$. The same larger value of the QT variance was observed during NIGHT in SYMP compared to ASYMP even though this SYMP-ASYMP difference was not significant likely due to the small size of the ASYMP and SYMP groups. This result is compatible with a more reactive sympathetic control even in presence of normal/elevated vagal drive during NIGHT and might account for the augmented risk of SYMP group when a sudden sympathetic stimulus might occur during NIGHT. On the contrary, the less important sympathetic drive in ASYMP subjects would produce a more 
favorable prognosis.

These results confirm that different autonomic states are associated to diverse risk profiles in LQT2 and underline the strong link between autonomic control and LQT2 [5,16], as already suggested for LQT1 $[6,17]$. Remarkably, the autonomic profile associated with the greatest risk in LQT2 appears to be different to that of LQT1 [16]: indeed, in LQT1 a more favorable prognosis is associated to a more reactive sympathetic control and a limited vagal response $[6,16,17]$. This result outlines the different role played by cardiac control in the two most common variants of LQTS [16] as well as the possibility of differentiating LQT1 and LQT2 groups using HP and QT variability markers above and beyond genotyping procedures [18].

\section{Conclusions}

This work carries out HP and QT variability analyses over 24h Holter ECG recordings with the aim at stratifying the arrhythmic risk in LQT2. We observed that having a longer HP, preserved circadian variations of the vagal control directed to the sinus node and a lower sympathetic modulation directed to the ventricles might be protective in LQT2, thus confirming the importance of the beta-blocker therapy also for this LQTS variant [1].

This study stresses the importance of assessing autonomic control in LQT2 based on HP and QT spontaneous variability. This analysis shows the different characteristics of cardiac control in LQT2 ASYMP and SYMP subjects and points out that different autonomic states are associated to diverse risk profiles, thus improving risk stratification in LQT2. We remark the need of elucidating the diverse role of autonomic control in LQT1 and LQT2 to better understand the pathophysiological mechanisms of LQTS.

\section{References}

[1] P. J. Schwartz and M. J. Ackerman "The long QT syndrome: a transatlantic clinical approach to diagnosis and therapy," Eur. Heart J., vol. 34, pp. 3109-3116, 2013.

[2] P. J. Schwartz and L. Crotti, "Long QT and short QT syndromes," In: Cardiac Electrophysiology. From Cell to Bedside, D. P. Zipes and J. Jalife Eds. 7th Edition, Elsevier, Philadelphia, PA, USA, pp. 893-904, 2018.

[3] M. E. Curran et al., "A molecular basis for cardiac arrhythmia: HERG mutations cause long QT syndrome," Cell, vol. 80, pp. 795-803, 1995.

[4] I. Goldenberg et al., "Beta-blocker efficacy in high-risk patients with the congenital long-QT syndrome types 1 and 2: implications for patient management," J. Cardiovasc. Electrophysiol., vol. 21, pp. 893-901, 2010.

[5] P. J. Schwartz et al., "Genotype-phenotype correlation in the long-QT syndrome: gene-specific triggers for lifethreatening arrhythmias," Circulation, vol. 103, pp. 89-95, 2001.
[6] A. Porta et al., "Autonomic control of heart rate and QT interval variability influences arrhythmic risk in long QT syndrome type 1," J. Am. Coll. Cardiol., vol. 65, pp. 367374, 2015.

[7] S. Akselrod et al., "Power spectrum analysis of heart rate fluctuation: a quantitative probe of beat-to-beat cardiovascular control," Science, vol. 213, pp. 220-222, 1981.

[8] A. Porta, E. Tobaldini, T. Gnecchi-Ruscone and N. Montano, "RT variability unrelated to heart period and respiration progressively increases during graded head-up tilt," Am. J. Physiol., vol. 298, pp. H1406-H1414, 2010.

[9] M. Baumert et al., "QT interval variability in body surface ECG: measurement, physiological basis, and clinical value: position statement and consensus guidance endorsed by the European Heart Rhythm Association jointly with the ESCWorking Group on Cardiac Cellular Electrophysiology," Europace, vol. 18, pp. 925-944, 2016.

[10] B. De Maria et al., "Concomitant evaluation of heart period and QT interval variability spectral markers to typify cardiac control in humans and rats," Front. Physiol., vol. 10, 2019, Art. no. 1478.

[11] A. Porta et al., "Performance assessment of standard algorithms for dynamic RT interval measurement: comparison between RTapex and RTend approach," Med. Biol. Eng. Comput., vol. 36, pp. 35-42. 1998.

[12] Task Force of the European Society of Cardiology and the North American Society of Pacing and Electrophysiology, "Heart rate variability. Standards of measurement, physiological interpretation, and clinical use.," Eur. Heart J., vol. 17, pp. 354-381, 1996.

[13] G. Baselli, A. Porta, O. Rimoldi, M. Pagani and S. Cerutti, "Spectral decomposition in multichannel recordings based on multi-variate parametric identification," IEEE Trans. Biomed. Eng., vol. 44, pp. 1092-1101, 1997.

[14] A. Porta et al., "Frequency domain assessment of the coupling strength between ventricular repolarization duration and heart period during graded head-up tilt," $J$. Electrocardiol., vol. 44, pp. 662-668, 2011.

[15] T. Noda et al., "Gene-specific response of dynamic ventricular repolarization to sympathetic stimulation in LQT1, LQT2 and LQT3 forms of congenital long QT syndrome," Eur. Heart J., vol. 23, pp. 975-983, 2002.

[16] L. Crotti et al., "Vagal reflexes following an exercise stress test. A simple clinical tool for gene-specific risk stratification in the long QT Syndrome" J. Am. Coll. Cardiol., vol. 60, pp, 2515-2524, 2012.

[17] V. Bari et al., "Multiscale complexity analysis of the cardiac control identifies asymptomatic and symptomatic patients in long QT syndrome type 1," PLoS ONE, vol. 9, 2014, Art. no. e93808.

[18] M. Viitasalo et al., "Differentiation between LQT1 and LQT2 patients and unaffected subjects using 24-hour electrocardiographic recordings," Am. J. Cardiol., vol. 89, pp. $679-685,2002$.

Address for correspondence.

Dr Vlasta Bari, PhD

IRCCS Policlinico San Donato - Department of Cardiothoracic, Vascular Anesthesia and Intensive Care

Via F. Fellini 4, 20097 San Donato Milanese, Milan, Italy

E-mail: vlasta.bari@grupposandonato.it 